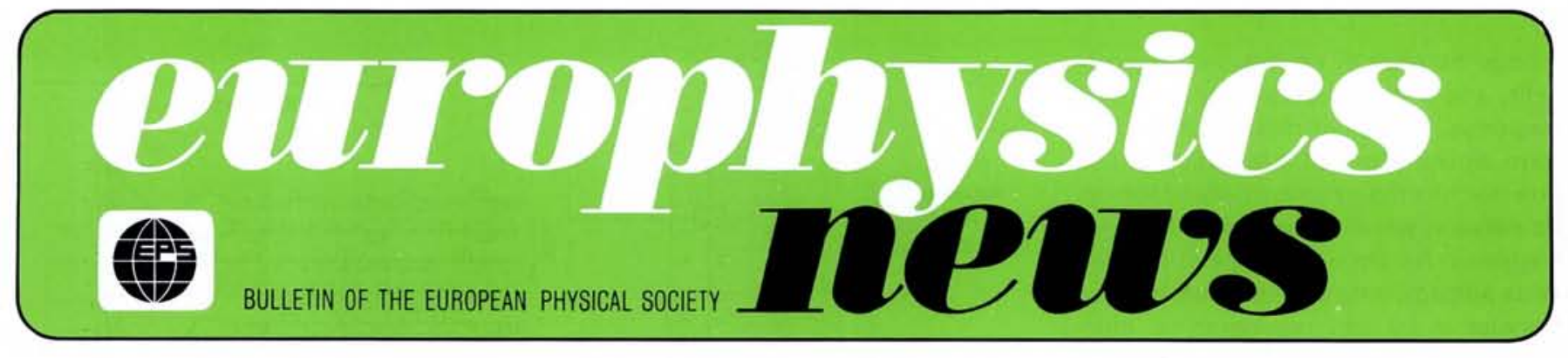

J.A.

Volume 16

Number 5

May 1985

\title{
From the Photon to the Neuronal Signal
}

\author{
Marc Chabre, Grenoble \\ (Laboratoire de Biophysique Moléculaire et Cellulaire, CEA-CENG)
}

Photons in the visible wavelength range, that is from 0.4 to $0.7 \mu \mathrm{m}$, provide the main source of information the brain receives from our physical environment. The maximal sensitivity of the visual system coincides with the maximum of the spectral distribution of sunlight photons reaching sea level, and is remarkably high: we are able to "see" one, or at least very few photons. From the few electron volts of energy of these photons the photoreceptor cells are able to generate a signal which is processed and transmitted to the brain. This implies a high amplification with perfect dark noise rejection. To a physicist the process may at first sight seem analogous to the rather simple photoelectric effect. It is in practice much more complex. It involves a large variety of phenomena, ranging from classical optics, molecular spectroscopy and photochemistry to enzymology and ion flux controls. Some of these are not very familiar to physicists. At the molecular level, these always reduce to quantum chemistry or classical physical chemistry of macromolecules. Rather than detailing one of these steps, I shall here describe the phenomenology and logic of the whole process.

The visual process may be divided into three main stages:

1. Optical imaging, to collect the photons scattered by objects in the visual field and focus them on the receptor cell layer of the retina.

2. Phototransduction through which the absorption of photons in the receptor cells leads to the generation of an electrical signal.
3. Neuronal processing, initiated in the retina and completed in the brain.

\section{Optical Imaging}

The physics of the optical system is fairly straight forward, at least in the eye of man and higher animals: a single lens, the crystalline, forms a two dimensional image of the complete visual field on the retina. In spite of its name, the transparent crystalline lens, is a concentrated but disordered "glass' type protein suspension, optically isotropic.

The optics is more complex in the insect compound eye which is constituted of an array of thousands of independent optical systems, each one subtending a very small solid angle. A high directivity for each elementary eye is insured by light guide properties of the receptor cell array behind each individual micro lens. No continuous optical image of the total field is ever made. The processing of the visual information in the insect "brain" must therefore be of a totally different type from that of the image processing in our brain. Here we shall restrict ourselves to the vertebrate eye.

The photoreceptor cells are the key element of the whole visual system, in which photon fluxes are converted into signals amenable to neuronal analysis. Except for some very primitive organisms, photoreceptor cells are not quantal "digital" counters, but rather proportional "analogue" transducers, which respond to increasing illumination by a progressive change of the electrical polarisation of their cellular membrane. The response is indeed quantal when less than one photon per second is ab- sorbed in a cell. It remains linear only at very low flux of illumination. The molecular mechanisms underlying this "photoelectric" conversion will be extensively discussed below. First, I shall sketch rather briefly the main features of the neuronal processing.

\section{Neuronal Processing}

This can again be divided into three main steps: analogue preprocessing in the retina, digitalisation and long distance transport in the optic nerve, and analysis in the brain before the totally unknown perception stage.

The retina is, embryologically and functionally an extension of the brain, where a significant analogue preprocessing is performed. So-called "horizontal cells" sense the polarisation changes on

\section{Contents}

From the Photon to the Neuronal Signal

EPS Meetings in Berlin-West Council

5th CMD General Conference

Applied Physics at the Condensed Matter Conference

Engineering Problems of a Thermonuclear Reactor

Letter to the Editor

Parity Violation Predictions

Danish Physics in a Steady State 15

Trends in Physics

16

Schools' News 
a large number of contiguous receptor cells, and sum and/or differentiate their response. Two other classes of cell perform similar types of integration before one reaches the ganglion cells of the optic nerve in which nervous impulses are triggered. As anywhere else in the nervous system, long distance information transfer is by self regenerating, standard-sized, nerve impulse "spikes" whose rate of firing codes the information. The retinal preprocessing allows the information collected by the $10^{8}$ photoreceptor cells of the retina to be transferred to the brain through only $10^{6}$ nerve fibres without loss of useful information. Only in a small central portion of the retina, involved in the highest resolution imaging, do photoreceptor cells benefit from a one to one connection with an optic nerve fibre. The angular resolution of vision is therefore not constant over the whole visual field. It is indeed fairly low except for a solid angle of a few degrees in the centre (fovea), which, by very fast and unconscious movements of the eye, we orient toward the details we want to observe. In the peripheral retina, lateral integration over a large number of cells enhances the absolute sensitivity by increasing the signal to noise ratio, at the expense of angular resolution. This retinal integration preserves however a very high sensitivity for the detection of movement of sources in the visual field.

Wavelength resolution, that is colour vision, is based on the analysis of the relative sensitivities to the light of a given wavelength of three types of photoreceptor cell (cone) differing in their spectral sensitivities. It is evident that the accurate determination of colours requires the absorption of large numbers of photons in each of the three types of receptor cells, to reduce the effect of statistical fluctuations in these numbers. Colour discrimination is therefore highly dependent on light intensity. Part of the colour coding is initiated in the retina, but most of the analysis is performed in the brain. The "colour" of an object is not in fact strictly defined by the spectral distribution of the photons it scatters. The processing involves comparisons with other parts of the visual field. This allows one to recognise a constant colour of an object observed under variable illumination conditions (Retinex theory).

Before arrival at the brain, the optic nerve fibres from both eyes cross in the optic chiasma where the fibres corresponding to the same part of the visual field in both eyes are sorted and directed towards the opposite side of the brain:

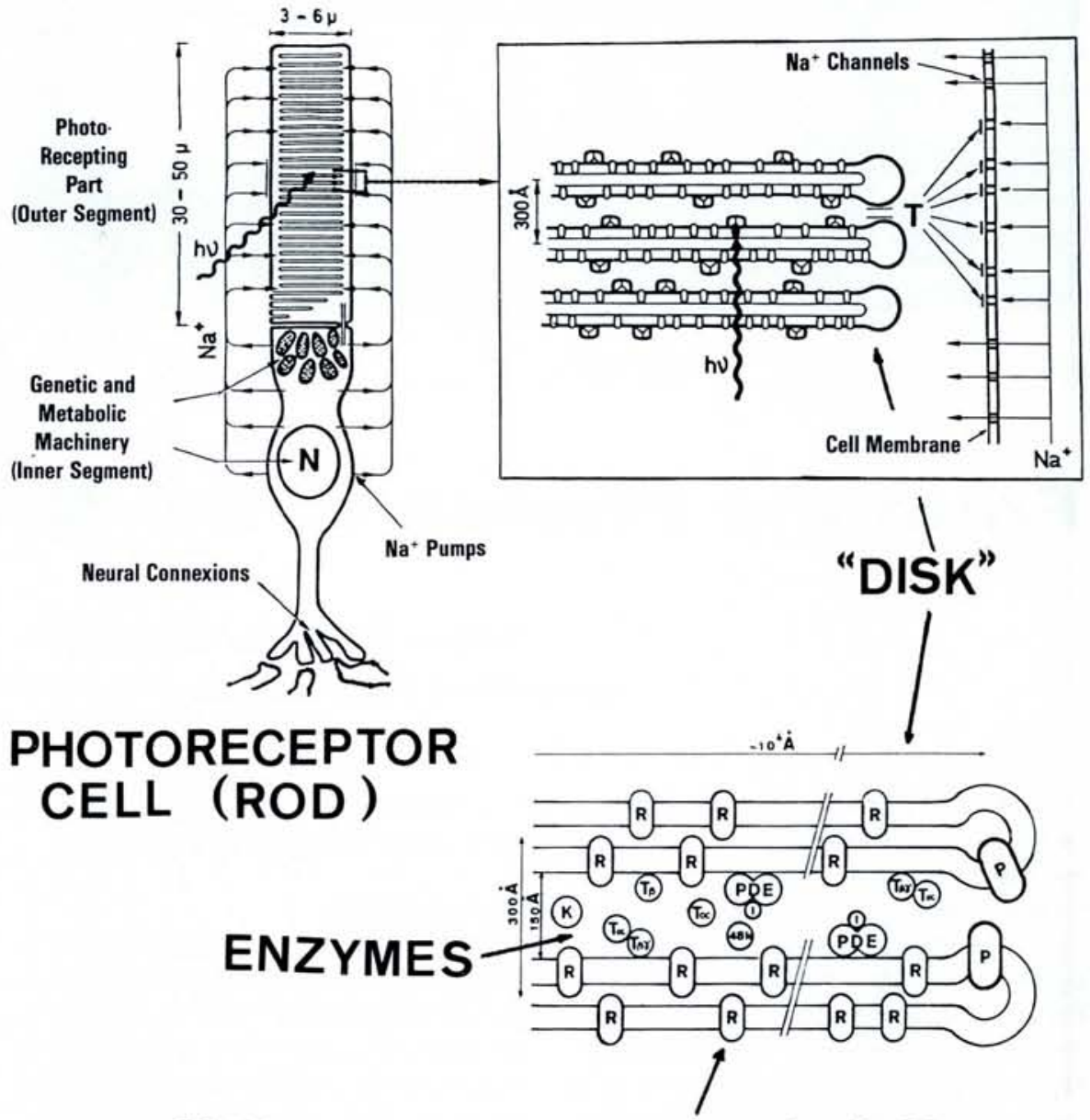

RETINAL

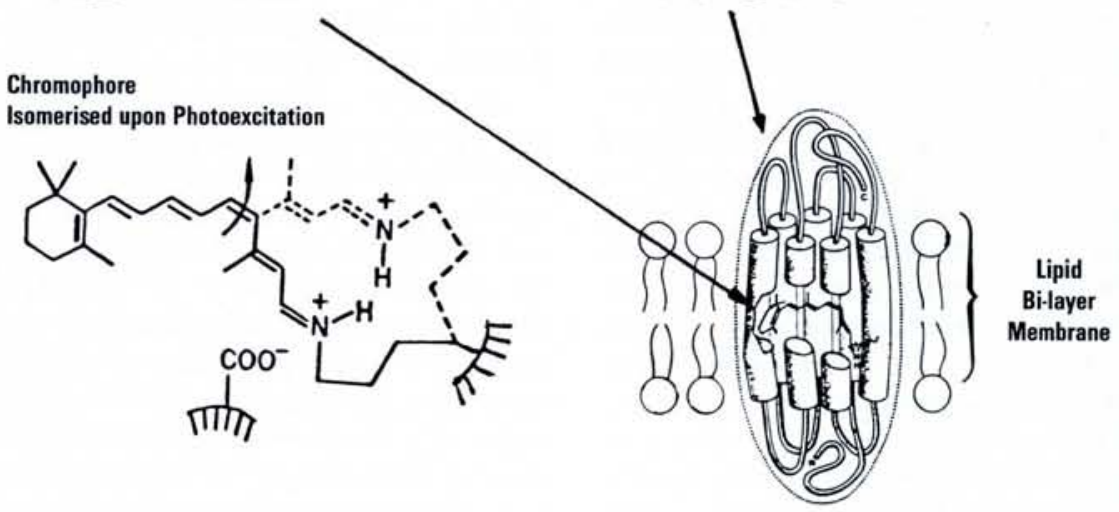

Fig. 1 - Structural and functional scheme of a photoreceptor cell. " $T$ " symbolises the soluble transmitter molecule (probably cGMP) which mediates the transmission of the message from the disk, where the photon is absorbed, to the cell membrane where the electrical signal is generated.

the left part of both retinas which receive the image of the right part of the visual field goes to the left part of the brain and vice versa.

The first steps of processing visual information in the brain have already been analysed in remarkable detail in particular by Hubel and Wiesel (Nobel 1981). The visual cortex where the first rudiments of pattern analysis are performed is by far the best known part of the brain cortex... but one is still very far from a deep understanding of visual perception.

\section{Phototransduction}

Between optical imaging and brain processing, the critical transduction stage has appeared surprisingly difficult to elucidate yet it is achieved with remarkable efficiency. A retinal rod cell has $90 \%$ efficiency for the absorption of 0.5 $\mu \mathrm{m}$ photons, and more than $60 \%$ quantum efficiency for the generation of a detectable signal by an absorbed photon. The process has a high gain, perfect dark noise rejection, and the capacity to adapt to an enormous intensity range: a light increment of $\sim 1 \%$ remains detec- 
table over 5 log units of light intensity. In compensation, responses may be slow and time resolution poor: near threshold intensity, that is below 1 photon/s, the electrical signal is delayed by a few tenths of a second after the photon capture. This is already indicative of the complexity of the intermediate mechanisms. The problem has attracted generations of scientists; many of them originated from physics. Simple and elegant models have been proposed, without much success yet. Indeed the concepts of physics appear less fruitful here than for example in photosynthesis. The collection of energy from photons in photosynthesis proceeds through molecular photoexcitation, exciton transfer and migration, trapping in reaction centres, energy storage by charge separation... many concepts that are familiar to photophysicists and solid state physicists. For phototransduction, the problem is not to collect energy, but information, and therefore to maximise signal over noise. The very first step is necessarily a molecular photoexcitation, where the photon energy is captured with high efficiency. But from then on, the event is memorized in a stable molecular conformation. This information must be transmitted to a cellular machinery which will generate a cellular response. Of this complex sequence of reactions, only the trigger and preamplification steps have been elucidated at the molecular level.

What is the electrical response of the cell? As in all cells, in photoreceptors an electrochemical potential difference is observed between the inside and the outside of the cell. It is due to selective ionic transport properties of the cell membrane, which result from the activity of "ion pumps" and "channels". The pumps are transmembrane proteins which, at the expense of energy that is provided by the concomitant hydrolysis of ATP (a high energy metabolite), extrude $\mathrm{Na}^{+}$outside the cell and concentrate $\mathrm{K}^{+}$ions inside. The channels are also transmembrane protein which provide passive but selective permeability to a given ion. $\mathrm{Na}^{+} / \mathrm{K}^{+}$pumps, also called ATPase in reference to their energy source, are found in all cells, but in the photoreceptor cells they are found only in the membrane of the inner segment (see Fig. 1) which contains exclusively the metabolic and genetic machinery; the membrane of the photoreceptive outer segment contains only $\mathrm{Na}^{+}$channels which are open in the dark. A Nernst equilibrium potential of $-30 \mathrm{mV}$ (inside/outside) results from the balance between the net influx of $\mathrm{Na}^{+}$in the outer segment, compensated by the active pumping from the inner segment. IIlumination causes the $\mathrm{Na}^{+}$channels of the outer segment to shut off. The Nernst potential is determined by the ratio of concentrations of permeant ions exclusively. Shutting off the $\mathrm{Na}^{+}$permeability results immediately in a decrease of the intracellular potential down to $-60 \mathrm{mV}$, corresponding to the ratio of inside/outside concentration of the other permeant ion $\mathrm{K}^{+}$. This potential gradient across the membrane is established over the whole surface of the cell membrane, and propagates in particular to the terminal "synaptic" region. In this region the potential gradient across the membrane controls the emission of chemical transmitters, which will in turn generate electrical signals in the apposed neuronal cells.

But, as seen in Fig. 1, in a retinal rod the photon is absorbed by a pigment molecule which is located in a membranous structure (the disc) topologically and electrically disconnected from the cell membrane. This excludes a priori any direct effect of the pigment molecule on the ionic conductance of the cellular membrane: an intracellular soluble transmitter, released or generated by the disc, must cross the aqueous medium to reach the membrane. Two candidates were proposed 15 years ago: the simple $\mathrm{Ca}^{++}$ion, a quite ubiquitous intracel!ular messenger, or a more complex molecule, cGMP which plays commonly a role in hormonal signalling. Intracellular transmitters are very difficult to work on, especially as is the case for $\mathrm{Ca}^{++}$, when the intracellular concentration is $10^{3}$ to $10^{4}$ times lower than the extracellular. It took about 15 years to get convincing evidence that $\mathrm{a} \mathrm{Ca}^{++}$ion storage existed within the disc and that most of it was released upon illumination - only to be followed by the conclusion that $\mathrm{Ca}^{++}$ions are not the excitatory transmitter: the kinetics of release does not coincide with that of excitation, which can be observed in the total absence of $\mathrm{Ca}^{++}$. The role of $\mathrm{Ca}^{++}$ is most probably in a slower adaptation process, by modulating the conductance of the $\mathrm{Na}^{+}$channel.

On the other hand, cGMP was long considered to be under the control of "slow" enzymatic reactions, but it has recently been established that indeed, light triggered a very fast amplifying cascade of enzymatic reactions controlling the destruction, hence the concentration of cGMP. How this reacts on the $\mathrm{Na}^{+}$channel conductance is not yet clear, but the mechanism of triggering, amplification and regulation of the

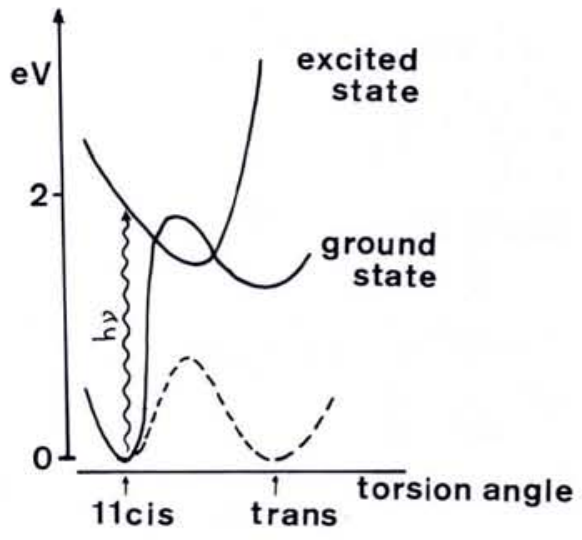

Fig. 2 - Potential energy barrier for the photoisomerisation of retinal in rhodopsin. The dotted line represents the same barrier for free retinal in solution.

cGMP signal are now quite well understood:

\section{The Trigger Mechanism}

The photon absorber is a highly conjugated polyene molecule, retinal, (a derivative of vitamine $A$ ), encaged within an insoluble membrane protein, rhodopsin (R). The gross structure of rhodopsin and its location within the disc membrane have been obtained only recently through the combination of very precise biochemical data, and the contributions of a large variety of biophysical approaches (X-ray and neutron diffraction, diamagnetic anisotropy, linear dichroism) which took advantage of the liquid crystal organization of the disc membrane in the rod cell. This was a small compensation for the fact that no real 3-D crystal of rhodopsin could be obtained, this membrane protein not being soluble in water, nor in any other solvent: a part of the molecule is hydrophilic and another part hydrophobic on a large area.

In the dark adapted state, a double bond of the retinal is in the "cis" conformation, which implies a bent polyene chain. The excited state reached upon photoexcitation has a high probability to relax down to the straight "all - trans" conformation of the polyene chain. For a retinal molecule that would be free in solution, the energy difference between the cis and trans conformers is very small ( $\cong 0.02 \mathrm{eV}$ ). A torsional energy barrier reduces however spontaneous isomerisation at room temperature. But in the protein, the retinal is not free and the specific interaction between the chromophore and the polypeptide chain has two major implications: 


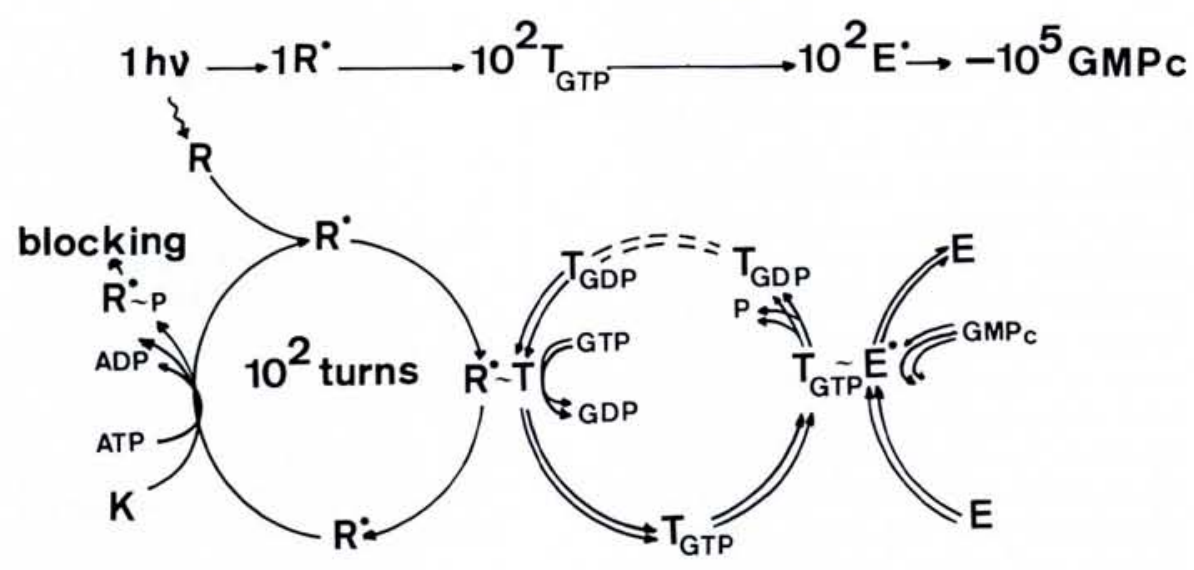

Fig. 3 - Amplification scheme through which one absorbed photon controls the hydrolysis of a large number of transmitter molecules.

a) - The "cis" ground state and the excitated state orbitals are perturbed in such a way as to shift the wavelength of maximal photon absorbance from the near UV to the visible range.

b) - The ground state energy level for the "all-trans" conformation of the bound retinal is shifted up by about $1 \mathrm{eV}$. Indeed the protein prevents a complete isomerisation of the retinal, which, after photoexcitation can reach only a "distorted all trans" conformation.

In this partial isomerisation, reached within picoseconds, $50 \%$ of the photon energy has remained stored as torsional energy. This introduces a high energy bias for the triggering of this reaction, which fully protects the system against thermal isomerisation $(k T \sim 0.02 \mathrm{eV}$ at room temperature). The stored energy will then force the protein structure to relax to adapt to the all-trans conformation of retinal. These structural changes will propagate much slower $\left(\sim 10^{-4} \mathrm{~s}\right)$ over the whole protein volume toward the protein water interface, until a new metastable state $R^{*}$, of long lifetime ( 10 s) which has developed catalytic sites on its hydrophilic surface is reached. The capture of a photon is now "memorised" in the existence of that metastable protein structure.

\section{Amplification: sequential memory reading}

The disc membrane is a fluid 2-dimensional layer in which $R^{*}$ as well as $R$ diffuse freely, with frequent collisions with other proteins bound to the same membrane. Among them, transducin $(T)$ has a high affinity for binding to $R^{*}$ (but not to $R$ ). Then $R^{*}$ acts as a catalyst on the bound $T$ and allows its "activation" by a triphosphate compound GTP, present in solution. These triphosphate compounds are a common energy source in biological systems. The hydrolysis of the third phosphate group is highly exoenergetic and when the compound is bound to a protein, this hydrolysis is usually coupled to an endoenergetic structural change of the protein. Once it has exchanged a "used up" diphosphate compound GDP, for a new GTP, transducin is released from $R^{*}$ as an active $T^{*}$. Note that, as a catalyst, $R^{*}$ has remained unchanged in this reaction. It will therefore be able to activate in sequence many $T$, at the rate of about $10^{3} / \mathrm{s}$, limited only by diffusion and collision coupling. This provides the first amplification stage. Now each $T^{*}$, again by diffusion encounters a third type of protein $E$, a hydrolytic enzyme specific of cGMP, which is active only when bound to $T^{*}$. Each $T^{*}-E$ complex catalyses the hydrolysis of cGMP (an exoenergetic reaction) at the rate of $\cong 10^{4} / \mathrm{s}$.

In this two step cascade, within 100 $\mathrm{ms}$, the approximate rise time of the visual signal, a single absorbed photon can induce the hydrolysis of about $10^{5}$ cGMP molecules. This is at the expense of more than $10^{4}$ times the photon energy.

\section{Regulation and Blocking}

Such a high gain amplification requires control, fast regulation and switch off mechanisms. Such a mechanism exists on $R^{*}$ : a soluble enzyme binds phosphate groups to $R^{*}$, then a second soluble protein will bind specifically to the phosphorylated $R^{*}$ : this blocks after a short delay the access of transducin to its catalytic site on $R^{*}$. This process is much slower than the blocked $R^{*}$ decay through which $R^{*}$ loses its chromophore and will be ultimately regenerated (memory reset) by binding a new "cis" chromophore.

A next step of regulation is on the $T^{*}-E$ complex, whose lifetime and efficiency are controlled by various factors, one of them being the hydrolysis of the GTP molecule which activated $\mathrm{T}$.

There certainly remain details to be worked out, but this basic scheme of triggering, preamplification and regulation is now solid. It is also highly probable that this same preamplification chain underlies both the excitation and the adaptation pathways.

\section{What next?}

Is cGMP directly interacting with the $\mathrm{Na}^{+}$channels on the cell membrane? This seemed unlikely, as cGMP is a common intracellular transmitter which, in all other known systems, acts through the activation of a rather complex series of activable enzymes. A very delicate technique allows one to pick up a small patch of cell membrane to seal the tip of a microelectrode. One can then study the conductance and sensitivity of its $\mathrm{Na}^{+}$channels to putative transmitters. In a very recent experiment, cGMP appeared directly active. One would then have the complete story for excitatory transmission, from the photoisomerization of retinal, to the closure of the $\mathrm{Na}^{+}$ channels. The "simple" last stage has, however, to be confirmed, and the molecular structure of the $\mathrm{Na}^{+}$channels remains totally unknown, one deals here only with functional entities. Whatever its mechanism, the last step of photoreceptor transduction bears a strong functional analogy with the final stage of a power amplifier: the signal results from cutting off a large current.

What about $\mathrm{Ca}^{++}$then? Its release from the discs seems to be under the control of a sequence of reactions that uses the same initial GTP and transducin cascade as the cGMP pathway; but the exact point of branching between the two pathways is not known. Intracellular $\mathrm{Ca}^{++}$can definitely modulate the $\mathrm{Na}^{+}$ channel conductance. The response to light being slower and less sensitive than that of the cGMP pathway, $\mathrm{Ca}^{++}$ ions have probably a major role in adapting the ionic fluxes in the cell under constant illumination.

\section{Conclusion}

This short glimpse of an apparently simple biophysical problem: how to signal the absorption of a photon, gives an idea of the intricacies of a real biological solution: the very strict constraint of environment and of "components" available in a biological cell exclude the use of physical phenomena commonly exploited in electronics. The cleverness of nature - or rather the success of evolution, is in the very efficient utilisation of very classical photochemical and biochemical reactions. 